\title{
Fulminanter Nutzenzuwachs in der Neurologie (Teil 2)
}

Hans H. Jung, Zürich; Christian Baumann, Zürich; Alain Kälin, Lugano; Kai Rösler, Bern; Andrea O. Rossetti, Lausanne; Kaspar Schindler, Bern; Michael Schüpbach, Bern; Margitta Seeck, Genf

In der Neurologie kam es in den letzten 20 Jahren zu grundlegenden, teils revolutionären Veränderungen der Behandlungskonzepte und -erfolge. Krankheiten wie Parkinson, Epilepsien und Polyneuropathien, die früher unbehandelbar waren, können nun mit neuen rationalen Therapiemethoden angegangen werden. Dies verbessert die Lebenserwartung und Lebensqualität der Betroffenen markant und reduziert Invaliditätskosten.

\section{Parkinson-Krankheit - länger selbständig leben}

Bewegungsstörungen betreffen in der Schweiz viele Patienten, alleine unter Parkinson leiden rund 15000 Personen, eine Zahl, die aufgrund der demographischen Entwicklung weiter ansteigen dürfte. Neben der häufigen Parkinson-Krankheit mit ihren fortschreitenden und stark beeinträchtigenden Symptomen wie Unbeweglichkeit, Steifheit, Zittern und Haltungsinstabilität gibt es viele weitere Bewegungsstörungen. So gehen Tremor-Erkrankungen mit unwillkürlichem Zittern einher, die oft verkannten Dystonien verursachen anhaltende und belastende Verkrampfungen und Fehlhaltungen, und viele genetische Erkrankungen, wie z.B. Erkrankungen des Kleinhirns, verursachen Störungen der Koordination.

Ein Paradebeispiel für den grossen Zuwachs an Therapiemöglichkeiten der letzten 20 Jahre ist die tiefe Hirnstimulation (THS), bei der durch die Implantation eines «Hirnschrittmachers» die Hirnfunktionen normalisiert und die Symptome gemildert werden. Dies hilft heute vielen Parkinson-, Tremor- und Dystonie-Patienten, deren Lebensqualität gesteigert wird und deren Arbeitsfähigkeit teilweise erhalten bleibt. Parkinson-Patienten profitieren heute auch oft vom Einsatz einer Pumpe, die ihre Medikamente direkt in den Darm abgibt und so einen gleichmässigen Medikamentenspiegel sicherstellt. Dies verbessert insbesondere den raschen Wechsel von Steifigkeit und Überbeweglichkeit und ermöglicht ihnen mehr Selbständigkeit. Auch das Vorgehen, mit Botulinumtoxin-Injektionen krankhaft überaktive Muskeln ruhigzustellen, hat sich etabliert und weiterentwickelt und verbessert vor allem die Beschwerden von Dystonie-Patienten effektiv und nachhaltig. Dank dieser und weiterer Fortschritte haben Patienten mit Bewegungsstörungen heute eine Lebensqualität, welche vor 20 Jahren undenkbar gewesen wäre.

\section{Epilepsien - den Sturm im Hirn in den Griff kriegen}

Für die 56000 an einer behandlungsbedürftigen Epilepsie erkrankten Menschen in der Schweiz - und die etwa 4000, die jedes Jahr hinzukommen - haben sich in den letzten 20 Jahren die Chancen auf eine erfolgreiche und verträgliche Behandlung und damit eine gute Lebensqualität signifikant erhöht. Ein Teil dieser Fortschritte beruht auf einer deutlichen Zunahme der verfügbaren antikonvulsiven Medikamente. Diese sind zwar nicht immer wirksamer, aber für viele Patienten deutlich besser verträglich als die «klassischen» Präparate. Auch haben sie deutlich geringere neuropsychologische Nebenwirkungen und eignen sich besser für Patientinnen mit einem Kinderwunsch.

Trotz der vielen neuen und verbesserten Medikamente erreichen jedoch immer noch rund 30\% aller EpilepsiePatienten medikamentös kein optimales Behandlungsergebnis. Für diese Patienten lässt sich heute deutlich besser feststellen, ob die umgrenzten Hirnregionen, von denen die epileptischen Anfälle ausgehen, chirurgisch entfernt werden können. Da die MRI-Bildgebung heute mit höheren Feldstärken (gemessen in Tesla) arbeitet, werden diese Herde viel besser entdeckt: Im 
Vergleich zu 1,5 Tesla werden mit 3 Tesla rund 50\% mehr Herde erkannt, mit 7 Tesla nochmals 30\% mehr. Auch durch die Analyse der Hirnaktivität mit dem neuen hochauflösenden Elektroenzephalogramm können epileptogene Herde genauer lokalisiert werden. Insgesamt können so mehr Patienten sinnvoll operiert werden und haben eine bis zu zehn Mal höhere Wahrscheinlichkeit, nach der Operation anfallsfrei zu werden. Falls eine chirurgische Entfernung der jeweiligen Hirnstrukturen nicht möglich ist, können Therapien wie die oben beschriebene THS oder eine VagusnervStimulation durch einen implantierten Schrittmacher angewendet werden. Dies führt zwar oft nicht zu Anfallsfreiheit, häufig jedoch zu einer markanten Reduktion der Häufigkeit oder Schwere der Anfälle.

\section{Polyneuropathie - besser leben dank massgeschneiderter Therapie}

Neuromuskuläre Erkrankungen umfassen sowohl Krankheiten der Muskeln (Myopathien) als auch Erkrankungen, bei denen deren Versorgung durch die Nerven gestört ist. Letzteres ist bei Krankheiten der peripheren Nerven (Neuropathien) und Krankheiten der die Muskeln steuernden Motoneurone (wie zum Beispiel der amyotrophen Lateralsklerose) der Fall. Neuromuskuläre Krankheiten betreffen viele Menschen. So schätzt man allein für die Polyneuropathien, dass rund 3\% aller über 60-Jährigen daran leiden.

Entzündliche Polyneuropathien wie das akute Guillain-Barré-Syndrom (GBS) beginnen meist mit Lähmungen an den Beinen und befallen innert einigen Tagen den ganzen Körper, so dass der Patient bei vollem Bewusstsein regungslos im Bett liegend auf umfassende Hilfe angewiesen ist. Unbehandelt kann ein solcher Zustand monate- bis jahrelang anhalten. Bei chronischeren Formen wie der CIDP treten die Lähmungen langsamer auf, verursachen aber auch langfristige Invalidität und Verlust der Selbständigkeit. Aufgrund der in den letzten beiden Jahrzehnten enormen Fortschritte bei Diagnose und Behandlung können diese Formen neuromuskulärer Erkrankungen heute aber zielgerichtet und effektiv angegangen werden. Durch elektrophysiologische und Blutuntersuchungen sowie MRI- und Ultraschall-Bildgebung können Subtypen dieser Polyneuropathien unterschieden werden, was gezieltere Behandlungen erlaubt. So kann beim GBS der Austausch von Blutplasma oder die Gabe konzentrierter Antikörper (intravenöser Immunglobuline, IvIG) das Fortschreiten der Lähmungen unterbrechen und oft eine rasche Rückbildung der Schwächen bzw. eine Heilung erreichen. Während GBS-Patienten früher häufig sehr lange im Spital oder Pflegeheim bleiben mussten und sich danach nicht völlig erholten, verlassen sie heute das Spital oft nach einigen Tagen und erlangen rasch ihre Arbeitsfähigkeit wieder. Bei der CIDP kann heute das früher gefürchtete Fortschreiten oft gestoppt werden. Dank klinischer und elektrophysiologischer Untersuchungen erhalten die Patienten dabei für sie bezüglich Dauer und Häufigkeit massgeschneiderte Therapien.

\section{Fazit: ein fulminanter Nutzenzuwachs}

Die Fortschritte in der klinischen Neurologie der letzten Jahre - hier dargestellt am Beispiel der ParkinsonKrankheit, der Epilepsien und der Polyneuropathien sind das Resultat einer intensiven Grundlagen- und klinisch-wissenschaftlichen Forschung und stellen eine Erfolgsgeschichte für die betroffenen Patienten dar. Für die Zukunft ist abzusehen, dass für viele bislang nicht kausal therapierbare neurologische Erkrankungen wie amyotrophe Lateralsklerose (ALS), Demenz oder genetisch bedingte neurologische und neuromuskuläre Erkrankungen spezifische Therapiemethoden zur Verfügung stehen könnten. Die Kosten dieser Entwicklung sind hoch, sind aber in einer Gesamtrechnung mit einer oft markant verbesserten Lebensqualität und verlängerten Lebenszeit zu sehen. Neben diesem Nutzen für die individuellen Patienten und ihr Umfeld erzielen die neuen Therapien auch einen ökonomischen Nutzen, weil oftmals z.B. Behinderungen und Berentungen vermieden oder verzögert werden und Pflegebedarf reduziert wird.

\section{Zusammenfassung}

Die Fortschritte der Neurologie haben in den letzten 20 Jahren zwar die Behandlungskosten erhöht, aber auch einen erheblichen Mehrwert für die Patienten gebracht. So profitieren Patienten mit Bewegungsstörungen heute von einer deutlich höheren Lebensqualität. Viel mehr Epilepsie-Patienten erreichen heute Anfallsfreiheit oder erhalten eine verträglichere Therapie, und Menschen mit entzündlichen Polyneuropathien erleben heute teilweise einen schnellen Rückgang oder zumindest einen Stopp des Fortschreitens der Symptome, wo früher langfristige Invalidität unvermeidbar war. Diese Fortschritte geben den Betroffenen Lebensqualität und Lebenszeit - und sparen der Gesellschaft, unter anderem, hohe Invaliditätskosten. 